\title{
A prescription for physical therapy and exercise in Parkinson's disease
}

\author{
Marie Saint-Hilaire, Theresa Ellis
}

School of Medicine, Boston University, Boston, USA; neuromsh@bu.edu

Received 15 August 2013; revised 16 September 2013; accepted 25 September 2013

Copyright (C) 2013 Marie Saint-Hilaire, Theresa Ellis. This is an open access article distributed under the Creative Commons Attribution License, which permits unrestricted use, distribution, and reproduction in any medium, provided the original work is properly cited.

The rate of progression of Parkinson's Disease (PD) varies among individuals living with the disease. However, there comes a time, later in the course of the disease, when most patients with PD develop balance impairments and falls. Unfortunately, these symptoms do not respond well to dopaminergic medications or deep brain stimulation. Typically, this is when the clinician caring for the patient initiates a referral to physical therapy (PT). There is accumulating evidence revealing the benefits of PT and exercise in reducing disability in persons with PD [1]; however, PT is often not recommended until the later stages of the disease when disability levels are substantial. The data from epidemiological studies randomized controlled clinical trials (RCT's) and studies in animal models of PD suggest that PT and engagement in exercise should be initiated early to reduce disability and optimize quality of life.

Although one early epidemiological study on the effect of exercise on the risk of developing PD failed to show a benefit [2], two larger prospective studies found a positive impact of exercise [3,4]. In the most recent prospective study of 213,000 participants in the NIH-AARP Diet and Health Study Cohort, a higher level of moderate to vigorous physical activity between ages 35 and 39, or in the past 10 years, was associated with a decreased risk of developing PD [3]. Individuals with consistent and frequent participation in moderate to vigorous physical activity in both periods had a $40 \%$ lower risk of developing PD compared to those who were inactive. This study was significantly larger than the previous ones, and data about exercise was uniformly collected.

Several clinical trials have shown that PT reduces disability in patients with PD. A recent Cochrane review identified 33 RCT's with 1518 subjects examining the benefits of PT compared to no-intervention in persons with PD [1]. Results revealed significant improvements in gait velocity, functional mobility, balance and activities of daily living following PT compared to no intervention. Improvements in quality of life, measured by the Parkinson Disease Questionnaire-39, were seen following a 6-week RCT using a self-management approach to rehabilitation which persisted at the 6-month follow-up visit [5]. This indicates the potential importance of helping people with PD learn the skills (i.e., the importance of regular exercise) they need to manage the disease over the long-term. Falls are common and potentially life threatening in PD, and there has been little evidence that exercise reduces falls [1]. However, a recent study investigating the benefits of 24 weeks of Tai Chi in patients with mild to moderate disease, showed significant improvements in balance, functional capacity, and reduced falls compared to resistance training and stretching conditions [6].

Studies in animal models of PD suggest several potential mechanisms to explain the beneficial effect of physical activity. These include secretion of neurotrophic factors, enhanced neurogenesis, angiogenesis, and neuroplasticity [7]. In a recent study of the 6-OHDA lesioned rat model of PD, treadmill exercise was found to significantly increase the number of tyrosine hydroxylase positive neurons in the lesioned substantia nigra, compared to the control group [8]. There was also significantly less muscle atrophy following exercise.

Another potential benefit of physical activity is its effect on cognition. Cognitive difficulties are frequently observed in PD as the disease progresses and people with PD have a six-fold increase in the risk of dementia [9]. A meta-analysis of prospective cohort studies revealed that physical activity reduced the risk of dementia by $28 \%$ in the general population, although this had not been demonstrated in a PD population [10]. A number of mechanisms have been proposed such as promotion of vascular health, decrease in inflammatory markers, and improvement of endothelial function.

Despite the evidence revealing the beneficial effects of exercise, studies reveal the profound inactivity among persons with PD. In a cross-sectional study comparing physical activity levels among persons with PD and 
older adults living in the community, persons with PD (n = 699) were found to be almost $1 / 3$ less active than age matched older adults $(n=1951)$ [11]. Levels of physical activity decreased with disease progression - a decline of 13\% from H\&Y 1 to 2 and 21\% from H\&Y 1 to 3 [11]. This highlights the need for initiation of an exercise program early in the disease. In a longitudinal study examining physical activity levels at baseline and one year later, results revealed that persons with PD walked $12 \%$ fewer steps and had a $40 \%$ reduction in the number of moderate intensity steps (>100 steps per minute) indicating a substantial decline in physical activity levels over a one year period [12]. A decline in walking ability has also been identified as a clinical "red flag" and a marker of emerging disability further emphasizes the need for early rehabilitation to optimize function and reduce disability [13].

Physical activity must be continued regularly for its benefit to be sustained [14]. Studies in persons with PD have identified low self-efficacy and outcome expectation as significant barriers to engaging in exercise [15, 16]. Programs to increase physical activity among persons with PD should include strategies to promote behavior change. The ParkFit Trial $(n=586)$ was a 2-year multicenter RCT comparing PT with a specific emphasis on promoting a physically active lifestyle (ParkFit Program) to matched PT with specific emphasis on safety and quality of performing ADL's (ParkSafe Program) [17]. The ParkFit program specifically focused on inducing a lasting change in exercise behavior through goal setting, overcoming barriers to engaging in exercise, recruiting social support, coaching by the PT, and use of an activity monitor with visual feedback regarding daily activity levels. Physical activity, measured using an activity monitor, revealed a significant increase in activity level $(12 \%, 95 \% \mathrm{CI}=7 \%-16 \%)$ over 24 months in the ParkFit condition compared to the ParkSafe condition [18]. Activity diaries also revealed an increase of approximately 90 minutes per week of physical activity compared to 30 minutes in the ParkSafe condition. No differences between groups were found using a physical activity questionnaire.

Innovative approaches (e.g., virtual coaches, dance programs) designed to meet the needs and preferences of individual patients with PD are important to facilitate sustained engagement in exercise in the community [19, 20]. Physical therapists (PT's) are uniquely positioned to design an exercise program tailored to the individual with PD with the goal of enhancing function and reducing disability. PT's can then refer patients with PD to community exercise programs that are appropriate given their level of function, individual preferences and goals. Regular follow-up visits with the therapist over the course of the disease allows for re-assessment of func- tion (i.e., gait, balance, moving in bed) and further tailoring of the exercise program to prevent or slow the onset of disability.

In conclusion, studies reveal profound sedentary lifestyles among persons with PD. There is a growing body of literature demonstrating the benefits of physical therapy and exercise in reducing disability in these patients. The animal literature suggests a potential disease modifying effect. For these reasons, PT should be part of the standard management of PD early in the course of the disease. Studies have shown that individual patient needs, preferences, and action directed toward realistic goals are fundamental to successful rehabilitation outcomes and should be considered to optimize long-term engagement in exercise.

\section{REFERENCES}

[1] Tomlinson, C.L., Patel, S., Meek, C., et al. (2012) Physiotherapy versus placebo or no intervention in Parkinson's disease. Cochrane Database of Systematic Reviews, 7, CD002817.

[2] Logroscino, G., Sesso, H.D., Paffenbarger, R.S. and Lee, I.M. (2006) Physical activity and risk of Parkinson's disease: A prospective cohort study. Journal of Neurology, Neurosurgery \& Psychiatry, 77, 1318-1322. http://dx.doi.org/10.1136/jnnp.2006.097170

[3] Xu, Q., Park, Y., Huang, X., et al. (2010) Physical activities and future risk of Parkinson disease. Neurology, 75, 341-348.

http://dx.doi.org/10.1212/WNL.0b013e3181ea1597

[4] Chen, H., Zhang, S.M., Schwarzschild, M.A., Hernan, M.A. and Ascherio, A. (2005) Physical activity and the risk of Parkinson disease. Neurology, 64, 664-669. http://dx.doi.org/10.1212/01.WNL.0000151960.28687.93

[5] Tickle-Degnen, L., Ellis, T., Saint-Hilaire, M.H., Thomas, C.A. and Wagenaar, R.C. (2010) Self-management rehabilitation and health-related quality of life in Parkinson's disease: A randomized controlled trial. Movement Disorders, 25, 194-204. http://dx.doi.org/10.1002/mds.22940

[6] Li, F., Harmer, P., Fitzgerald, K., et al. (2012) Tai chi and postural stability in patients with Parkinson's disease. The New England Journal of Medicine, 366, 511-519. http://dx.doi.org/10.1056/NEJMoa1107911

[7] Ahlskog, J.E. (2011) Does vigorous exercise have a neuroprotective effect in Parkinson disease? Neurology, 77, 288-294. http://dx.doi.org/10.1212/WNL.0b013e318225ab66

[8] Choe, M.A., Koo, B.S., An, G.J. and Jeon, S. (2012) Effects of treadmill exercise on the recovery of dopaminergic neuron loss and muscle atrophy in the 6-OHDA lesioned Parkinson's disease rat model. The Korean Journal of Physiology \& Pharmacology, 16, 305-312. http://dx.doi.org/10.4196/kjpp.2012.16.5.305

[9] Aarsland, D., Andersen, K., Larsen, J.P., Lolk, A., Nielsen, H. and Kragh-Sorensen, P. (2001) Risk of dementia in Parkinson's disease: A community-based, prospective study. 
Neurology, 56, 730-736. http://dx.doi.org/10.1212/WNL.56.6.730

[10] Hamer, M. and Chida, Y. (2009) Physical activity and risk of neurodegenerative disease: A systematic review of prospective evidence. Psychological Medicine, 39, 3-11. http://dx.doi.org/10.1017/S0033291708003681

[11] van Nimwegen, M., Speelman, A.D., Hofman-van Rossum, E.J., et al. (2011) Physical inactivity in Parkinson's disease. Journal of Neurology, 258, 2214-2221. http://dx.doi.org/10.1007/s00415-011-6097-7

[12] Cavanaugh, J.T., Ellis, T.D., Earhart, G.M., Ford, M.P., Foreman, K.B. and Dibble, L.E. (2012) Capturing ambulatory activity decline in Parkinson's disease. Journal of Neurologic Physical Therapy, 36, 51-57. http://dx.doi.org/10.1097/NPT.0b013e318254ba7a

[13] Shulman, L.M., Gruber-Baldini, A.L., Anderson, K.E., et al. (2008) The evolution of disability in Parkinson disease. Movement Disorders, 23, 790-796. http://dx.doi.org/10.1002/mds.21879

[14] Haskell, W.L., Lee, I.M., Pate, R.R., et al. (2007) Physical activity and public health: Updated recommendation for adults from the American College of Sports Medicine and the American Heart Association. Medicine \& Science in Sports \& Exercise, 39, 1423-1434. http://dx.doi.org/10.1249/mss.0b013e3180616b27

[15] Ellis, T., Cavanaugh, J.T., Earhart, G.M., et al. (2011) Factors associated with exercise behavior in people with Parkinson disease. Physical Therapy, 91, 1838-1848. http://dx.doi.org/10.2522/ptj.20100390
[16] Ellis, T., Boudreau, J.K., Deangelis, T.R., et al. (2013) Barriers to exercise in people with Parkinson disease. Physical Therapy. http://dx.doi.org/10.2522/ptj.20120279

[17] van Nimwegen, M., Speelman, A.D., Smulders, K., et al. (2010) Design and baseline characteristics of the ParkFit study, a randomized controlled trial evaluating the effectiveness of a multifaceted behavioral program to increase physical activity in Parkinson patients. BMC Neurology, 10, 70 .

[18] van Nimwegen, M., Speelman, A.D., Overeem, S., et al. (2013) Promotion of physical activity and fitness in sedentary patients with Parkinson's disease: Randomised controlled trial. $B M J, 346$, f576. http://dx.doi.org/10.1136/bmj.f576

[19] Ellis, T., Latham, N.K., Deangelis, T.R., Thomas, C.A., Saint-Hilaire, M. and Bickmore, T.W. (2013) Feasibility of a virtual exercise coach to promote walking in community-dwelling persons with Parkinson disease. American Journal of Physical Medicine \& Rehabilitation, 92 472-485.

[20] Foster, E.R., Golden, L., Duncan, R.P. and Earhart, G.M. (2013) Community-based Argentine tango dance program is associated with increased activity participation among individuals with Parkinson's disease. Archives of Physical Medicine and Rehabilitation, 94, 240-249. 